\title{
Guidelines for cytogenetic investigations in tumours
}

\author{
Rosalind J Hastings ${ }^{1}$, Nick Bown ${ }^{2}$, Maria G Tibiletti ${ }^{3}$, Maria Debiec-Rychter ${ }^{4}$, Roberta Vanni ${ }^{5}$, Blanca Espinet ${ }^{6}$, \\ Nadine van Roy $^{7}$, Paul Roberts ${ }^{8}$, Eva van den Berg-de-Ruiter ${ }^{9}$, Alain Bernheim ${ }^{10}$, Jacqueline Schoumans ${ }^{11}$, \\ Steve Chatters ${ }^{12}$, Zuzana Zemanova ${ }^{13}$, Marian Stevens-Kroef ${ }^{14}$, Annet Simons ${ }^{14}$, Sverre Heim ${ }^{15}$, Marta Salido ${ }^{6}$, \\ Bauke Ylstra ${ }^{16}$ and David R Betts ${ }^{\star, 17}$
}

European Journal of Human Genetics (2016) 24, 6-13; doi:10.1038/ejhg.2015.35; published online 25 March 2015

Cytogenetic and molecular genetic data are of paramount importance in the diagnosis, prognosis, and risk stratification of patients with malignant diseases. Sometimes they even directly guide the choice of therapy. ${ }^{1}$ Disease-specific abnormalities, particularly translocations, can provide essential information to assist the Pathologist and/or Oncologist in assigning a diagnosis. In several diseases, tumour genetics correlate strongly with clinical risk; thus, cytogenetic information may help the Oncologist counsel the patient, choose a specific treatment, and/or modulate treatment intensity. Clinical trials may involve cytogenetic classification of patients to the appropriate treatment regimens.

Currently, the provision of specific assays for acquired neoplasiaspecific genomic changes varies among and within countries as a range of laboratories offer diagnostic solid tumour genetics; these may include Cytogenetic, Pathology, Haematology, and Molecular Genetics laboratories. Technical standards and general guidelines for the analysis and the report of results on most solid tumours are lacking.

To address these deficits, a tumour best practice meeting with invited tumour experts without conflict of interest was held on 23rd April 2013 in Oxford, United Kingdom. The aim was to produce professional guidelines for tumour genetic laboratories and to incorporate the standards imposed by generic European guidelines, ${ }^{2}$ regulatory bodies (ISO15189, 2012 Medical laboratories - requirements for quality and competence), ${ }^{3}$ reporting guidelines, ${ }^{4}$ ISCN, ${ }^{5}$ and acquired best practice guidelines, while taking into account the current practice in Europe.

The guidelines are aimed principally at giving guidance on the minimum, standard cytogenetic analyses, which are applicable to different types of laboratories operating under different regulatory arrangements and are relevant if more specific recommendations are not available. It was universally acknowledged that information on ancillary techniques in use in most cytogenetic laboratories (eg, RT-
PCR) or advanced techniques not always extensively performed in all laboratories (eg, next-generation sequencing (NGS)) were considered.

The process for developing these evidence-based consensus guidelines included agreement on the need of general uniform rules on solid tumour analysis and reporting, discussion on the architecture of the guidelines, working group formation with different tasks (collection, analysis and comparison of any existing guidelines on this subject, type of tumours to be included according to published data and database consultation, method of analysis to be included, report formulation), circulation of the working group activities, formulation and circulation of the initial recommendations, draft and discussion, final consensus, and approval.

It is noted that some elements of the tumour diagnostic service not subject to statute may be varied according to local constraints and agreements. Therefore, these guidelines are minimum requirements and additional professional judgment may be of paramount importance under many circumstances. In addition, as new techniques, particularly NGS, as well as clinical evidence, are becoming available all the time, these should be kept under constant review.

Notes: The use of 'must' in this document indicates a requirement and the use of 'should' indicates a recommendation. Where there appears to be contradiction between available guidelines, the most recently published ones should be taken to apply to all. All diagnostic laboratories should be accredited to national or internationally accepted standards (ISO15189). ${ }^{3,6}$ Laboratories should participate in an External Quality Assessment Scheme ${ }^{7}$ in all aspects of their service for which a scheme is available.

\section{LABORATORY STAFFING}

The laboratory must have either a head of laboratory or a senior staff member who is knowledgeable in the cytogenetic abnormalities, the appropriate test(s) required, and the clinical significance of results for

\footnotetext{
${ }^{1}$ Women's Centre, John Radcliffe Hospital, Oxford University Hospitals NHS Trust, Oxford, UK; ${ }^{2}$ Northern Genetics Service, Institute of Genomic Medicine, Central Parkway, Newcastle, UK; ${ }^{3}$ UO Anatomia Patologica, Ospedale di Circolo-Polo Universitario, Varese, Italy; ${ }^{4}$ Laboratory for Genetics of Malignant Disorders, Department of Human Genetics, University Hospital Gasthuisberg, UZ Leuven, Leuven, Belgium; ${ }^{5}$ Department of Biomedical Sciences, University of Cagliari, Cittadella Universitaria, Monserrato, Italy; ${ }^{6}$ Laboratori de Citogenètica Molecular, Servei de Patologia, Hospital del Mar, Barcelona, Spain; ${ }^{7}$ Centre for Medical Genetics, University Hospital Ghent, Ghent, Belgium; ${ }^{8}$ Cytogenetics Department, St James's Hospital, Leeds, UK; ${ }^{9}$ Department of Genetics, University of Groningen, University Medical Centre Groningen, RB Groningen, The Netherlands; ${ }^{10}$ Génétique des tumeurs (INSERM U985), Laboratoire de Cytogénétique, Pathologie Moléculaire Gustave Roussy, Paris-Villejuif, France; ${ }^{11}$ Cancer Cytogenetic Unit, Lausanne University Hospital, CHUV, Lausanne, Switzerland; ${ }^{12}$ Haematology, Cellular and Molecular Diagnostic Service, Great Ormond St Hospital, London, UK; ${ }^{13}$ Center of Oncocytogenetics, Institute of Clinical Biochemistry and Laboratory Diagnostics, General University Hospital and First Faculty of Medicine, Charles University in Prague, Prague, Czech Republic; ${ }^{14}$ Radboud University Nijmegen Medical Centre Department of Human Genetics, HB Nijmegen, The Netherlands; ${ }^{15}$ Institute for Cancer Genetics and Informatics, The Norwegian Radium Hospital, Oslo University Hospital, Oslo, Norway; ${ }^{16}$ Department of Pathology, Cancer Center Amsterdam, VU University Medical Center, MB Amsterdam, The Netherlands; ${ }^{17}$ Department of Clinical Genetics, Our Lady's Children's Hospital, Crumlin, Dublin, Ireland

*Correspondence: DR Betts, Department of Clinical Genetics, Our Lady's Children's Hospital, Crumlin, Dublin 12, Ireland. Tel: +353 14096738 ; Fax: +353 14096971 ; E-mail: david.betts@olchc.ie
}

Received 6 June 2014; revised 19 January 2015; accepted 3 February 2015; published online 25 March 2015 
all tumour types that the laboratory may process. Appropriately trained staff members analysing these tumours should be familiar with the reason for the test and findings that are non-random in that tumour type.

\section{COMMUNICATION}

It is strongly recommended that the laboratory performing the cytogenetic analysis has a close liaison and dialogue with the referring Pathologist and/or Oncologist both pre- and postsample receipt to gain information regarding the quality of the specimen received, its tumour cellularity, and the suspected tumour type. As the processing and analysis of tumour samples may be time-consuming and expensive, updates on the working diagnosis will allow the most effective directing of work, for example, facilitating the provision of a rapid and most appropriate fluorescence in situ hybridisation (FISH) test.

Subsequent prompt communication regarding the nature of the sample allows for effective work flow and prioritisation, for example, it may prevent the analysis of samples, which turn out to be reactive or non-neoplastic. Second, histological information on the nature of the specimen, in particular the tumour cell content, is often essential to interpret chromosome abnormalities, FISH, and NGS results.

\section{SOURCES OF MATERIAL}

Tumour analysis may be carried out using fresh, frozen, fixed, formalin-fixed paraffin- embedded (FFPE) tissue or using cytological material (fine-needle aspirates (FNAs)) or dissociated cells or tissue. In some instances, infiltrated bone marrow material can be used. Methods for processing of tumour material will be determined by the cytogenetic laboratory based on available clinical and pathologic findings. In instances where fresh viable material is obtained, the cytogenetic laboratory should seek as much information as possible about the differential diagnosis and the tissue type at the time of sample receipt to choose the most appropriate processing techniques.

The amount of material received, typically in the form of a tumour biopsy, can vary greatly, for example, whether it derives from an open surgical procedure or a needle biopsy. In the near future, bodily fluids such as urine, faeces, blood, and sputum would be expected to be reliably suitable for the cytogenetic analysis of solid tumours.

FISH analysis can be carried out on fixed, frozen, FFPE, FNA, or touch preparations (TPs) from fresh tumour tissue. These specimens will be the principal target for FISH, as they will most closely represent the cell populations in the tumour biopsy unlike cultured cells for banding analysis, which may not represent the tumour parenchyma tissue.

Fresh, frozen, FFPE, and, in some instances, TPs from fresh tumour tissue material can also be used for DNA extraction for subsequent microarray ${ }^{8}$ or NGS testing. ${ }^{9,10}$

For chromosome banding analysis, the tissue sample must be fresh and ideally without necrosis. It is essential to collect the tumour sample under sterile conditions and to select a representative area or areas of the tumour to improve success rate; therefore, the laboratory should have procedures in place whereby fresh tissue can be transported and processed promptly. Many laboratories provide sterile culture medium to local surgical units or pathology departments for this purpose, and although sterile saline may also be used, it is considered inferior to the culture medium. Tumour biopsies sent for banding analysis must not be frozen or fixed before dispatch to the cytogenetic laboratory as cells are no longer viable following these processes.
The laboratory must have clear guidelines on any subsequent retention of patient material postdiagnostic testing. ${ }^{11}$

\section{Paraffin-embedded tissue}

Formalin-fixed, paraffin-embedded tissue is acceptable. Tumour sections should be cut $3-4 \mu \mathrm{m}$ thick and mounted on positively charged organosilane-coated (silanized) slides. Before analysis of FFPE section slides, a pathologist should review a haematoxylin- and eosinstained (H\&E) slide and delineate the region of tumour cells that should be examined because it can be difficult to differentiate normal cells from malignant cells using only DAPI counterstain. The analyst should know, before scoring the slide, where the malignant cells of interest are located on the slide. For microarray and NGS analysis, enrichment of malignant cells by macroscopic dissection of the tumour cells on the slides is beneficial for the results.

\section{Touch preparations}

TPs are helpful when tissue architecture is not crucial. In most instances, a pathologist and/or operating oncologist will make the TP or be involved in selecting the tissue for TP. TPs should be made by lightly touching a tumour piece to a dry glass slide without smearing; for larger biopsies, using flat cut surface will produce optimal results. Subsequent preparation of these slides, before FISH analysis, may be laboratory or tumour specific. If the laboratory receives pre-made slides, rather than preparing them within the laboratory, there should be communication about how the slides should be made, how many are required, and subsequently sent. The laboratory should have a system to evaluate the received slides and whether they are appropriate for the test required.

\section{Cytospin preparations}

Cytospin preparations are useful for concentration of samples with very low cellularity, for example, cerebrospinal fluid and urine. These preparations should be prepared rapidly following acquisition of the sample and care must be taken to ensure that the cell morphology remains intact, for example, by using a slow spin speed.

\section{Fresh-frozen tumour tissues}

Such tissues may be useful in sequential analysis of recurring tumours or in the evaluation of archived specimens.

\section{Fine-needle aspirates}

Such samples are sometimes used especially in paediatric oncology, as fine-needle aspiration is minimally invasive and usually provides high amount of tumour cells, particularly in round-cell tumours.

Fixed cytogenetically prepared cells

Such preparations have multiple uses for both interphase and metaphase FISH evaluations, including confirmation and clarification of suspected chromosome abnormalities or characterisation of an apparently abnormal clone.

\section{SAMPLE PREPARATION}

Each laboratory should have written details of how it processes each sample when it is received. It is also important that a strategy is in place to determine the order of importance of techniques in instances where there is limited amount of material.

\section{Fresh tissue}

This must be evaluated and processed rapidly. Where possible, presumed viable tumour material should be separated from 
non-viable material, for example, calcified, or non-tumour material such as fat. Disaggregation of the tumour material can be performed either enzymatically and/or mechanically, for example, with scalpels. The choice of technique may depend on biopsy size and presumed tumour type.

\section{Archival material, for example, slides}

The laboratory must ensure that the material on slides is received in such a way that subsequent analysis is not compromised, for example, stained or marked by felt tip or diamond marker. Depending on result urgency, the slides may be immediately preprocessed, either in the form of fixation or pepsin treatment for FISH analysis. Otherwise, slides may be stored either at -20 or $4{ }^{\circ} \mathrm{C}$ in the case of FFPE slides, until required.

\section{TECHNIQUES}

For many tumour types, often a single cytogenetic technique will not generate all clinically relevant results; there are a number of other techniques that are frequently used in conjunction with cytogenetic methods that further aid in the determination of the genetic makeup of the tumour. Individual techniques have strengths and weaknesses and the choice of test may ultimately depend on local expertise, relationship to the clinical and pathology departments, published recommendations, and typical size and form of sample. Nevertheless, all laboratories should have an awareness of the advantages and disadvantages of each technique and how they may complement each other. The latter consideration is an important factor in the final interpretation of the cytogenetic features of a tumour.

Table 1 is a non-exhaustive list of the major tumour types currently investigated, together with the results that should be obtained by a laboratory and a preferential method by which results are obtained.

\section{Fluorescence in situ hybridisation}

In many instances, FISH is used as a stand-alone technique, for example, testing for a gene fusion and has the advantage that can be used on archived material as FISH does not require cells in division (see Appendix). In addition, it can be used in conjunction with chromosome banding to aid karyotype characterisation.

The technique is applicable to a range of material sources, including:

- Tumour touch imprint slides prepared from fresh or frozen tumour samples.

- FFPE sections.
- Intact cells released from paraffin blocks, which are dropped onto slides or used in cytospin preparations.

- Infiltrated bone marrow.

- Other infiltrated tissue, for example, ascites or cerebrospinal fluid.

- Urine.

- Cultured harvests of fresh tumour tissue*.

${ }^{*}$ If cultured preparations are used, these ideally should be either directly harvested or from short-term cultures ( $72 \mathrm{~h}$ maximum). Longer-term cultures should only be used if it is known that there are tumour parenchyma cells present and, in most instances, as an attempt to further characterise an abnormality that is known to be present.

The advantage of using touch imprint slides or paraffin sections is that an H\&E-stained slide can be prepared from the same cut surface, allowing for assessment of tumour cell content by the Pathologist.

Rapid results can be achieved, often within $24 \mathrm{~h}$, when necessary. It is frequently the method of choice to detect fusion products, and fusion products and gene amplification.

Reporting negative results from analysis of infiltrated bone marrow or other tissue requires particular consideration. As a result of haemodilution, the proportion of tumour cells in the sample sent to the laboratory can be much lower than that reported by the Haematologist, or bone marrow aspirates may be taken from multiple sites and may show widely differing levels of tumour infiltration. A further consideration is that some hospitals will define the presence of bone marrow infiltration as determined by a trephine investigation; therefore, the diagnostic laboratory must be aware on what basis bone marrow infiltration has been defined.

Although the technique is less labour intensive and requires less training than banding analysis, complex signal patterns and cell-to-cell variation is common in solid tumour samples, thus skilled personnel are required to ensure accurate reporting. FISH analysis is not a genome-wide test and can only answer questions specifically related to the probe(s) used. Interphase FISH analysis is a suitable method to investigate genetic heterogeneity in solid tumours; however, in situations where multiple probes are used, it rapidly becomes expensive.

\section{Chromogenic in situ hybridisation/silver-enhanced in situ hybridization}

Chromogenic in situ hybridization/silver-enhanced in situ hybridization (CISH/SISH) is an alternative to FISH on FFPE slides, which can be analysed using a brightfield microscope. ${ }^{12}$ These techniques have

Table 1 Current method of choice for required tests associated with specific tumour types

\begin{tabular}{|c|c|c|c|}
\hline Tumour type & Test required & Method of choice & Other non-essential tests and methods \\
\hline Breast carcinoma & $E R B B 2(H E R 2)$ & FISH (dual-colour)/CISH/SISH & Microarray, NGS \\
\hline Carcinoma & Disease-specific rearrangement & FISH (if available probes) or RT-PCR & Banding, microarray, NGS \\
\hline Lipomatous tumours & Subtype-specific changes & FISH, banding & Banding, MLPA (dosage) \\
\hline Neuroblastoma & MYCN status & FISH (dual-colour) & $\begin{array}{l}1 p, 11 q, 17 q \text { status (FISH or MLPA), banding, microarray, } \\
\text { NGS }\end{array}$ \\
\hline Gliomas & $1 p, 19 q$ status & FISH, or qPCR, or MLPA & Microarray, NGS, banding \\
\hline Other CNS tumours & $\begin{array}{l}\text { MGMT methylation; disease- or grade-specific } \\
\text { changes }\end{array}$ & FISH (eg, MYC in medulloblastoma) & $\begin{array}{l}\text { Banding, MGMT methylation analysis (MS-MLPA, MS-PCR, } \\
\text { pyrosequencing), microarray }\end{array}$ \\
\hline Renal tumours & Subtype-specific changes & Banding & Microarray, FISH \\
\hline Soft tissue sarcoma & Sarcoma-specific fusion product & $\begin{array}{l}\text { FISH (if available probes), } \\
\text { or RT-PCR, banding }\end{array}$ & Banding \\
\hline Wilms tumour & & & Banding, FISH, microarray \\
\hline
\end{tabular}


the advantage of allowing evaluation of target/gene status simultaneously with tissue morphology. As the labelling is permanent, longterm archiving is possible. The technique is limited to one or two colours, and the turnaround time is generally slower than for FISH. Usually this technique is used for gene amplification detection and it is not an appropriate test to detect other chromosome abnormalities. As with FISH, skilled personnel are required to ensure accurate reporting.

\section{Microarray (SNP/oligo)}

Microarray analysis is applicable to any tumour type and is being increasingly used in a diagnostic setting, for example, in neuroblastoma and clonality assays. Single-nucleotide polymorphism (SNP) arrays provide information about both chromosomal copy number and loss of heterozygosity (LOH) (including copy number neutral LOH), ${ }^{13}$ but few SNP array techniques perform well with FFPEderived DNA for diagnostic purposes. ${ }^{8}$ In contrast, CGH arrays cannot detect copy number neutral LOH, but can be reliably used with FFPEderived DNA. In addition to a 'pangenomic' overview, microarray analysis allows detection of very small regions of loss or gain/ amplification $^{14}$ and, in the case of SNPs, regions of LOH and a good indication to the ploidy of the tumour cells can be deduced.

Microarray analysis can also provide a more exact determination of breakpoints, which is valuable in larger series of individual tumours to determine potential clinical and/or biological significance of specific events. However, this technique it is not a suitable method to identify balanced rearrangements, including gene fusions, and genetic heterogeneity.

For array analysis, it is important that DNA is extracted from a region with high tumour cell content ( $>30 \%$ tumour cells). ${ }^{15}$ The technique can be sensitive to DNA quality, with low-quality DNA leading to failed tests or increasing the likelihood of calling false positives. Knowledge of tumour ploidy level is important and the determination of secondary-type events is difficult.

\section{Chromosome banding analysis}

It is well recognised that obtaining tumour metaphases for banding analysis is technically challenging. Increasingly alternative cytogenetic/ molecular techniques may be more appropriate as direct harvests or short-term cultures $(<72 \mathrm{~h})$ often provide no metaphases and longterm cultures are prone to overgrowth by non-tumour cells. If the laboratory receives sufficient tumour material, cultures for chromosome analysis should be initiated, as successful banding can provide much diagnostically helpful information, including: insights into translocations and chromosome partners in unbalanced events; copy number abnormalities (CNAs); and ploidy-level information and evidence of tumour cell heterogeneity. Chromosomal banding is starting to be superseded by NGS for the determination of clonal evolution and whether this is linear or divergent. ${ }^{16}$ Chromosome banding analysis can also clarify atypical or unusual FISH patterns, which would otherwise be hard to interpret. Furthermore, as a 'pangenomic' overview, banding analysis is open to unexpected results, which may lead to sudden changes in diagnostic direction. This may also include the detection of a constitutional abnormality.

This technique requires fresh material and will only provide information on the cells in division. In many instances, only longerterm cultures, in which the overgrowth of fibroblasts is a frequent event, will provide metaphase spreads for analysis. Banding analysis is relatively labour intensive and requires skilled, trained personnel. Tumour-related karyotypes are frequently complex, which may lead to many unidentifiable chromosomal regions and/or misidentification of chromosomes (particularly with undertrained personnel), and does not provide information on zygosity. In most instances, it is not possible to achieve a result within $24-48 \mathrm{~h}$ and frequently the report time would be measured in days or weeks.

Hence, while often highly informative, chromosome analysis can be unreliable as a sole approach, and all laboratories offering a solid tumour service must have access to supplementary techniques.

\section{Multiplex ligation-dependent probe amplification}

Multiplex ligation-dependent probe amplification (MLPA) can give an indication of imbalances and CNAs of the loci that are included in the reaction kit. Commercial MLPA kits are available to detect relevant CNAs in, for example, neuroblastoma, oligodendroglioma, and breast cancer. In addition, the technique can be used to investigate methylation status of significant genes such as MGMT in high-grade gliomas.

MLPA requires only small quantities of DNA, which should be extracted from materials with high tumour content. The method is fast, cheap, and very simple to perform. The regions tested are defined by the kit used and balanced translocations cannot be detected. MLPA analysis in tumour samples could be inaccurate because of genomic instability, the presence of several genetic alterations, and contamination with normal DNA. The technique is not useful to investigate genetic heterogeneity.

\section{Flow and static cytometry of total DNA content}

This technique will indicate the tumour ploidy. It provides an accurate determination of the DNA content of tumours and may prove valuable when used as an adjunctive technique in association with microarray and, occasionally, FISH. However, it should not be the first method of choice for tumour cytogenetic analysis and is not advised when there is only limited material available.

\section{SUPPLEMENTARY TECHNIQUES \\ RT-PCR}

Reverse transcriptase (RT)-PCR is frequently used as first-line technique to identify gene fusions resulting from translocations, particularly in the diagnosis of sarcomas, when fresh or frozen material is available. It has the advantage of being able to be designed as a multiplex technique, screening a number of known rearrangements in a given tumour group, for example, round-cell tumours. RTPCR can often clarify unusual or equivocal cytogenetic or FISH results by confirming or excluding key gene fusions and can be used to confirm the partner gene in the event of a positive breakapart FISH probe result. It is recommended that cytogeneticists involved in solid tumour analysis should liaise closely with appropriate units offering RT-PCR analysis, to define the order of use of the two types of techniques.

\section{Specific sequencing}

Sanger sequencing (SS) can detect all base substitutions, small insertions, and deletions, but has a modest limit of detection, which can be highly variable depending on the exact sequence, and the laboratory performing the test. Using an automated interpretation algorithm with a $10 \%$ threshold, SS yielded $11.1 \%$. The limit of mutation detection by SS is subjective and may depend on the experience level of the person interpreting the data.

Pyrosequencing (PS) is a bioluminescence technique in which the pyrophosphate released during incorporation of a nucleotide into a growing DNA chain is converted to light through a series of enzymatic reactions. PS can identify individual bases or short stretches of nucleic acid sequence at predetermined positions. 
Tumour cell heterogeneity in conjunction with the fact that all specimens will contain a percentage of non-tumour cells may result in a relatively low percentage of mutated alleles within some specimens.

\section{NGS of DNA}

NGS allows for the analysis of mutations, DNA copy number variation, $\mathrm{LOH}$, balanced and unbalanced translocations, ${ }^{17}$ and methylation. ${ }^{18}$

Genome-wide, high-resolution NGS for copy number analysis performs well with small amounts of tumour material. The technique is suitable for DNA isolated from fixed, frozen, FFPE, FNA, or TPs from fresh tumour tissue and bodily fluids. ${ }^{19}$ The digital nature of the data lowers the ambiguity of interpretation as clear cutoffs can be set for diagnostic/clinical purposes. However, some bioinformatics skills maybe required and the large amount of data provides both computational and data storage challenges. ${ }^{20}$ In addition, the offtarget information may pose ethical questions that need to be addressed. ${ }^{21}$

Increasingly, next-generation, genome-wide sequencing and/or targeted sequencing is used in the routine diagnostic workup of solid tumours. As this is a young technique, the validation and implementation trajectory still requires special attention regarding both sensitivity and specificity of these techniques before use in diagnostics for solid tumor testing. ${ }^{22,23}$ The technique seems robust, but hardware infrastructure, operation system, software stack, and the particular version of these put novel challenges to the cytogenetic diagnostic practice. Thus, although we may be at a pivotal point in the history of cytogenetics for solid tumours, describing NGS guidelines for solid tumours seems premature, as this would be based on the experience of only few certified laboratories.

\section{FOLLOW-UP SAMPLES}

The choice of technique may also depend on the disease status and whether the sample is posttreatment, relapse, or a potential metastasis. In most instances, the laboratory should choose a technique that will either help to demonstrate the presence of tumour cells or the emergence of a known prognostic/clinical marker. In most instances, FISH would be the method of choice.

\section{SUCCESS RATES}

The success rate will depend on the technique and the quality and quantity of material received.

The fresh tumour samples received in cytogenetic laboratories can be very variable in amount and quality. Samples may be small needle cores to large surgical biopsies and vary widely in terms of tumour content, viability, and necrosis. Taking sample variation and the diversity of tumour types encountered into account, it is unrealistic to specify target success rates for banded chromosome analysis.

In contrast, interphase FISH for the detection or exclusion of specific aberrations is consistently reliable and the laboratory should aim for a success rate of $>95 \%$.

In a diagnostic setting, a laboratory should not rely on a technique, or combined techniques, that gives an informative success rate of $<90 \%$.

\section{REPORTING TIMES}

The clinical significance of cytogenetic input and the urgency with which it is required is highly variable from one case to the next, even within a given disease type, and it will depend to a great extent on the Pathologist's confidence in the results from other tests such as immunohistochemistry to provide an unequivocal diagnosis.
Currently, the number of cytogenetic markers that will directly influence treatment decisions is limited, but this is expected to change over time. If the test is known to influence treatment decisions, then the testing laboratory must be aware of any time requirement within the treating protocol and report within that time frame. In the absence of a specific treatment protocol, where cytogenetics could have a bearing on diagnosis and/or treatment, efforts should be made to report all results within 14 days. Otherwise, unless specified, it would be realistic to expect a final result within 28 days.

\section{ANALYSIS AND CHECKING}

For all analysis types, two analysts, one being a registered clinical scientist, or equivalent, must be involved in the analysis or checking of all diagnostic samples. In every case, a suitably qualified person must confirm that appropriate investigations have been carried out at an acceptable level of quality with respect to the referral reason.

\section{FISH}

The laboratory should define the number of individual cells that should be scored. For interphase FISH, a minimum of 100 individual cells is recommended. This number may need to be increased, particularly in instances where tumour content is unknown and low numbers of cells with an apparently abnormal signal pattern are seen. Where possible, separate cells should be scored, but if there are overlapping cells that cannot be scored individually, the tumour cell percentage must be known.

The laboratory should define its own cutoffs for individual FISH probes and tissue/sample types. For this purpose, they should also take into account the probe manufacturer's guidelines. For FFPE material, consideration must be given to the potential for truncated cells and the percentage of tumour cells in the section as defined by the pathologist. Laboratories must also be aware of the definition of imbalance and amplification for particular tumour types and genetic markers, for example, the definition of amplification may be tumour specific. ${ }^{24-26}$ Care should be taken in describing deletion/duplication in cells with polysomy. The use of control probes when assessing gain/loss is strongly recommended.

\section{Microarray}

The laboratory should define their minimum DNA-quality criteria, minimum quality parameters as defined by the system used and the minimum reportable size of an event. ${ }^{15}$ The latter aspect may also be modified depending on gene content of the region involved. Analysis and checking should involve at least two trained analysts.

\section{Banding}

The morphology of tumour metaphase chromosomes may be inferior to that of normal cells and it is important to examine metaphase spreads of varying quality until an abnormal clone is detected. Agreement on abnormal clones should be reached by two analysts. It is recommended that a minimum of 10 metaphase spreads are analysed if a clone is defined and 20 metaphases if no clone is identified. However, given the low mitotic index for some tumours, it is acceptable to analyse fewer than 10 cells if there are sufficient abnormal cells to establish the presence of an abnormal clone. If analysing posttreatment material or long-term cultures only, consideration must be given to the possibility of treatment-induced or culture-only events. 


\section{REPORTING}

The reporting of tumour cytogenetic results should be concise and unambiguous, with the result and written description to include sufficient detail to give the referring clinician and/or pathologist a clear understanding of the results. The individual reporting the results should have appropriate training, experience and qualification and may be a scientist or a clinician depending on National requirements. The report should include the following:

- The type of sample, including details of whether fresh, fixed, or frozen.

- The site of origin of sample, where appropriate.

- The test result(s) and written description.

- A clear statement of whether the result is normal or abnormal, and banding results should be recorded in the report using the current version of ISCN, including cell numbers.

- FISH analysis results should be recorded in the report using current ISCN or by using unambiguous language to describe the result and should include:

- A clear FISH result summary, if abnormal, is essential.

- The number of cells analysed should be given.

- Actual numbers of cells examined should be given, rather than percentages.

- An unambiguous statement in the summary result if a number of different clones are present in the sample. Details of the type and manufacturer of all FISH probes.

- Limitations of the test used, especially in the event of a normal result.

- A brief description of clinically relevant abnormalities.

- The clinical significance of result, if applicable, in relation to referral reason.

- The relationship of any abnormalities found to the referral reason, or other possible diagnoses.

- The association with prognosis or risk group if a robust association from multiple publications/international trials/ trial protocols exists.

- A comment if the gene rearrangement detected has also been reported in other tumour types (if differential diagnosis).

- For arrays: Type of array/platform; genome reference build number; an estimate of the tumour cell percentage; minimum resolution of platform and what is the minimum size of event reported.

- Use the current array ISCN where appropriate. It is appreciated that ISCN may be difficult for tumour arrays, and therefore an alternative unambiguous presentation of array results would be acceptable.

- If FFPE FISH, identification of specific block number on report (eg, Section B of 6).

- Cross-references to other tests as appropriate.

In instances where multiple cytogenetic tests are performed, a laboratory should issue a summary report in which a combined interpretation of all tests is made.
Where abbreviated cytogenetic results are integrated into a multidisciplinary report, the information in the abbreviated version should be consistent with the full cytogenetic report. The abbreviated cytogenetic summary shall be authorised by a state-registered clinical scientist or equivalent. A full version of the cytogenetic report must be sent independently to the referring clinician.

It is recommended that the term 'malignancy' is avoided particularly in the context of an abnormality of unknown significance. Terms such as 'clonal proliferation' or 'neoplasm' are recommended instead.

Normal karyotypic results in particular must always be regarded with suspicion, and these reports must include caveats regarding the likelihood of analysis of non-tumour cells, if analysed from long-term cultures, or analysis of reactive cells if analysed from short-term cultures.

Similarly, if interphase FISH results are normal (negative), then consideration should be given to the potential tumour cell content of the sample analysed; the potential clinical significance of the negative results should be discussed promptly with the referring clinician and/or pathologist. When reporting negative FISH results, knowledge of tumour cell content in the material analysed should be regarded as essential. If the tumour content of the tested material is unknown or in doubt, reports of negative results must be strongly qualified.

Normal results from DNA-based methods should consider whether the DNA has been extracted from tumour-rich material.

The laboratory should have a policy of the issuing of preliminary or verbal reports in instances of the detection or exclusion of an important clinical marker while other testing is still ongoing, without delaying the final report.

If a potential constitutional abnormality is detected, analysis of a PHA-stimulated blood sample may be appropriate. This may require an onward referral to a constitutional cytogenetic laboratory. There should be consultation with the patient's clinician and reference the potential need for genetic counselling for either the patient or their family.

Laboratories should follow their guidelines for sign-off of the report; however, it is recommended that at least one signature is a staff member of senior grade who is defined as competent to analyse and interpret tumour cytogenetic results.

\section{CONFLICT OF INTEREST}

The authors declare no conflict of interest.

\section{ACKNOWLEDGEMENTS}

We wish to acknowledge feedback from Barbara Dewaele, Belinda Carleer, Jérome Couturier, Vanessa Vanspauwen, and other colleagues in their departments on these guidelines. We would also like to acknowledge the contribution of Alain Bernheim who sadly passed away before the publication of these guidelines. These guidelines resulted from a Tumour Best Practice meeting held on 23rd April 2013 in Oxford that that was funded by Eurogentest (FP7- HEALTH-F4-2010-261469). We thank Cheryl Guiver for her help in the organisation of the Best Practice meeting.

\footnotetext{
1 Heim S, Mitelman F (eds): Cancer Cytogenetics: Chromosomal and Molecular Genetic Aberrations of Tumor Cells, 3rd edn. New York: Wiley-Blackwell.

2 European Cytogenetic Guidelines: ECA Newsletter, January 2012. Available at: http://e-c-a.eu/files/downloads/E.C.A._General_Guidelines_Version\%202.0.pdf (last accessed 16 January 2015).

3 ISO 15189:2012 Medical laboratories-Requirements for quality and competence.

4 Claustres M, Koz'ich V, Dequeker E et al: on behalf of the ESHG Quality committee: Recommendations for reporting results of diagnostic genetic testing (biochemical, cytogenetic and molecular genetic) (2013). Eur J Hum Genet 2013; 22: 160-170.
} 
5 Shaffer LG, McGowan-Jordan J, Schmid M: ISCN 2013: An International System for Human Cytogenetic Nomenclature. Basel: S Karger, 2013.

6 European co-operation for accreditation: Available at: http://www.european-accreditation.org/ (last accessed 16 January 2015)

7 UK NEQAS Executive Office: Sheffield S10 2PB. Available at: http://www.ukneqas.org.uk (last accessed 16 January 2015).

8 Krijgsman 0, Israeli D, Haan JC et al: CGH arrays compared for DNA isolated from formalin-fixed, paraffin-embedded material. Genes Chromosomes Cancer 2012; 51: 344-352.

9 Sie D, Snijders PJ, Meijer GA et al: Performance of amplicon-based next generation DNA sequencing for diagnostic gene mutation profiling in oncopathology. Cell Oncol (Dordr) 2014; 37: 353-361.

10 Tuononen K, Mäki-Nevala S, Sarhadi VK et al: Comparison of targeted next-generation sequencing (NGS) and real-time PCR in the detection of EGFR, KRAS, and BRAF mutations on formalin-fixed, paraffin-embedded tumor material of non-small cell lung carcinoma-superiority of NGS. Genes Chromosomes Cancer 2013; 52: 503-511.

11 The Royal College of Pathologists: The Retention and Storage of Pathological Records and Archives. London: The Royal College of Pathologists, 2006.

12 Peiró G, D Mayr DHillemanns $P$ et al: Analysis of HER-2/neu amplification in endometrial carcinoma by chromogenic in situ hybridization. Correlation with fluorescence in situ hybridization, HER-2/neu, p53 and Ki-67 protein expression, and outcome. Mod Pathol 2004; 17: 277-287.

13 Van Loo P, Nilsen G, Nordgard SH et al: Analyzing cancer samples with SNP arrays. Methods Mol Biol 2012; 802: 57-72.

14 Krijgsman O, Carvalho B, Meijer GA, Steenbergen RD, Ylstra B: Focal chromosomal copy number aberrations in cancer-Needles in a genome haystack. Biochim Biophys Acta 2014; 1843: 2698-2704

15 Krijgsman O, Israeli D, van Essen HF et al: Detection limits of DNA copy number alterations in heterogeneous cell populations. Cell Oncol (Dordr) 2013; 36: 27-36.

16 McGranahan N, Swanton C: Biological and therapeutic impact of intratumor heterogeneity in cancer evolution. Cancer Cell 2015; 27: 15-26.
17 de Vree PJ, de Wit E, Yilmaz M et al: Targeted sequencing by proximity ligation for comprehensive variant detection and local haplotyping. Nat Biotechnol 2014; 32 : 1019-1025.

18 Watson IR, Takahashi K, Futreal PA, Chin L: Emerging patterns of somatic mutations in cancer. Nat Rev Genet 2013; 14: 703-718.

19 Scheinin I, Sie D, Bengtsson $\mathrm{H}$ et al: DNA copy number analysis of fresh and formalin-fixed specimens by shallow whole-genome sequencing with identification and exclusion of problematic regions in the genome assembly. Genome Res 2014; 24: 2022-2032.

20 Ding L, Wendl MC, McMichael JF, Raphael BJ: Expanding the computational toolbox for mining cancer genomes. Nat Rev Genet 2014; 15: 556-570.

21 Rigter T, Henneman L, Kristoffersson $U$ et al: Reflecting on earlier experiences with unsolicited findings: points to consider for next-generation sequencing and informed consent in diagnostics. Hum Mutat 2013; 34: 1322-1328.

22 Ulahannan D, Kovac MB, Mulholland PJ, Cazier J-B, Tomlinson I: Technical and implementation issues in using next-generation sequencing of cancers in clinical practice. Br J Cancer 2013; 109: 827-835.

23 Tripathy D, Harnden K, Kimberly Blackwell K, Robson M: Next generation sequencing and tumor mutation profiling: are we ready for routine use in the oncology clinic? BMC Med 2014; 12: 140 (http://www.biomedcentral.com/1741-7015/12/140/_ins1).

24 Ambros PF, Ambros IM, Brodeur GM et al: International consensus for neuroblastoma molecular diagnostics: report from the International Neuroblastoma Risk Group (INRG) Biology Committee. Br J Cancer 2009; 100: 1471-1482.

25 Walker RA, Bartlett JM, Dowsett M, Ellis IO et al: HER2 testing in the UK: further update to recommendations. J Clin Pathol 2008; 61: 818-824.

26 Wolff AC, Hammond MEH, Hicks DG et al: Recommendations for human epidermal growth factor receptor 2 testing in breast cancer: American Society of Clinical Oncology/College of American Pathologists Clinical Practice Guideline Update. J Clin Oncol 2013; 31: 3997-4014. 


\section{APPENDIX}

Commercially available FISH probes that may assist in cytogenetic investigation of specific tumours

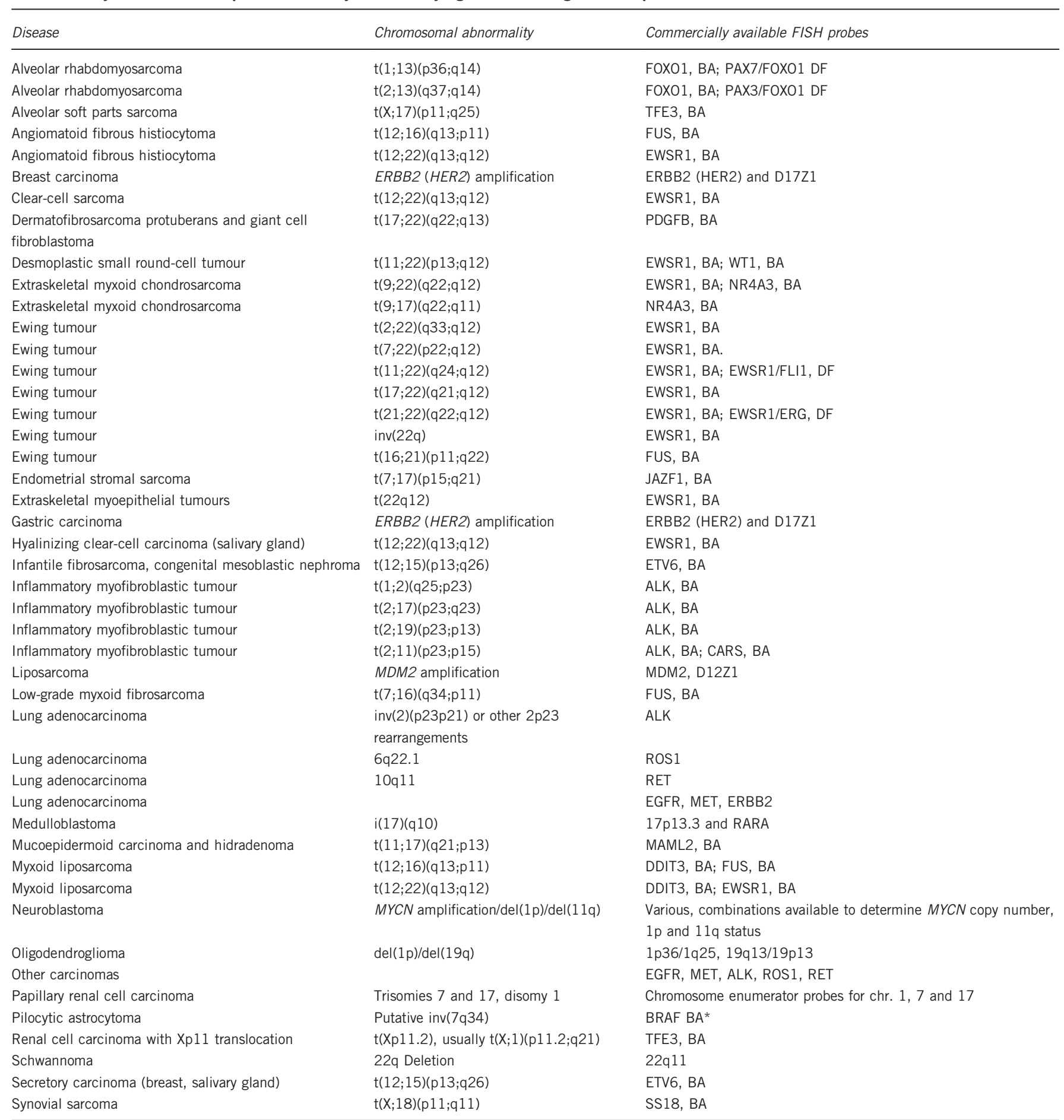

Abbreviations: BA, break apart; DF, dual fusion.

${ }^{*} B R A F$ activation through the KIAA1549-BRAF fusion has also been described in other paediatric low-grade gliomas (eg, pilomyxoid astrocytoma). BRAF point mutations (V600E) are observed in non-pilocytic paediatric low-grade gliomas as well, including approximately two-thirds of pleomorphic xanthoastrocytoma cases and in ganglioglioma and desmoplastic infantile ganglioglioma. 